\title{
Salud Pública: Objeto de Conocimiento, Prácticas y Formación ${ }^{1}$
}

\author{
Public health: knowledge, practice and training \\ Edgar C. Jarillo-Soto y Oliva López-Arellano
} Universidad Autónoma Metropolitana-Xochimilco, México. jsec6322@correo.xoc.uam.mx,
oli@correo.xoc.uam.mx

Recibido 28 Septiembre 2006/Enviado para Modificación 18 Enero 2007/Aceptado 8 Febrero 2007

\section{RESUMEN}

Se presenta una discusión sobre los principales retos que enfrenta la salud pública en torno a su objeto de conocimiento, como práctica profesional y como espacio de formación de personal. Se parte del reconocimiento del contexto socio-sanitario desigual y polarizado de América Latina y de un conjunto de referentes particulares para el desarrollo del campo sanitario, entre los que destacan las Funciones Esenciales en Salud Pública, los Objetivos y Metas del Milenio y la Asociación Latinoamericana de Educación en Salud Pública. Se exponen tres componentes que interesa destacar, el objeto de conocimiento (niveles, dominios, disciplinas, temporalidad, dimensiones y complejidad), la práctica social (público-estatal, práctica general de los colectivos y práctica profesional de grupos) y la formación de personal (profesional, técnica, así como capacitación y actualización) y en cada uno de ellos en forma breve se señalan cuales son los desafíos que se consideran esenciales. Se concluye con un conjunto de propuestas para ser impulsadas desde la Asociación Latinoamericana de Educación en Salud Pública: intercambio, cooperación y abogacía.

Palabras Clave: Educación en salud pública, conocimiento en salud pública, práctica en salud pública (fuente: DeCS, BIREME).

\section{ABSTRACT}

This paper presents a discussion regarding Public Health's main challenges: knowledge of it, professional practice and training human resources. It begins by recognising Latin-America's unequal and polar socio-sanitary context and the sanitary field's myriad single referents, paying special attention to essential public health functions, the Millennium development goals and the Latin-American Association of Public Health Education. Emphasis is placed on three components: knowledge of public health (levels, domains, disciplines, temporality, dimensions and complexity), social practice (state-public, collective general practice and group professional

${ }^{1}$ Este artículo se basa en el trabajo presentado en la XXI Conferencia de la Asociación Latinoamericana y del Caribe de Educación en Salud Pública (ALAESP), Río de Janeiro, 19-20 de agosto de 2006. 
practice) and human resources' training (professional, technical, training and updating). An essential challenge is then identified for each of these components and a set of proposals to be launched from the Latin-American Association of Public Health Education is outlined (interchange, partnerships and advocacy).

Key Words: Public health education, knowledge of public health, public health practice (source: MeSH, NLM).

$\mathrm{P}$

lantear el futuro de la salud pública implica considerar aspectos del pasado y del presente, a partir de su diferenciación como campo de conocimiento que ha evolucionado desde la detección de factores de de riesgo inmediato y biológico en la producción de las enfermedades, hasta la explicación de los determinantes producto de las formas de organización de las sociedades contemporáneas. En ese trayecto, la explicación de la enfermedad ha sido la constante, por lo que Granda (1) la refiere como "enfermología pública", lo que implica que más allá de sus aportes en la búsqueda de la salud, el eje explicativo dominante continúa siendo la enfermedad. Transitar a una explicación científica de la salud como objeto de conocimiento sigue siendo una deuda pendiente.

Con relación al objeto de estudio, hoy pocos actores cuestionan la confluencia de las dimensiones de la existencia humana marcada por sus rasgos orgánicos de orden biológico, incluso desde el nivel genómico, hasta las dimensiones sociales más generales que se expresan en distintas magnitudes y niveles de daños a la salud entre los individuos de un país, región o comunidad. Esta confluencia muestra un campo de conocimiento donde se debate sobre la naturaleza y formas de representación de la salud y de la enfermedad. Así, la comprensión del objeto de estudio remite a sus características materiales y simbólicas e individuales y colectivas, aún cuando las relaciones e interacciones entre ambas dimensiones estén insuficientemente exploradas.

Además, la salud pública se conforma como práctica a través de la intervención del Estado y remite a circunstancias de especificidad histórica. Evidencia de lo anterior, es la constante búsqueda de respuestas explicativas de la forma como participan los profesionales de la salud en las acciones sanitarias que implican su práctica. Un aspecto relevante ha sido la formación de la fuerza de trabajo que actúa en el campo de la salud pública en consonancia con las necesidades de salud en un espacio y tiempo delimitado, las intervenciones técnicas individuales y los programas masivos, y especialmente la participación en situaciones de emergencia donde se agudizan los problemas o se hace evidente la dificultad de instrumentar la acción colectiva. 


\section{LOS REFERENTES}

El contexto general que enmarca el pensar y el hacer de la salud pública en América Latina, se caracteriza por la desigualdad social y económica, la polarización socio-sanitaria y la pobreza generalizada.

Para el 2000, los indicadores generales de empleo, salario y distribución del ingreso de las economías latinoamericanas, muestran una región con niveles elevados de pobreza y creciente polarización social y económica. Según información de la CEPAL el porcentaje de pobres crece en América Latina de $41 \%$ (1980) a 44 \% (2000). En el mismo sentido, el informe 2004 del Banco Mundial señala que en 1981 sobrevivían 36 millones de personas en América Latina con un ingreso diario de un dólar (pobres extremos), cifra que se incrementa a 50 millones para el año 2001. Mientras que los latinoamericanos que viven con dos dólares por día pasaron de 99 millones en 1981 a 128 millones en 2001 (2). Respecto a la apropiación de la riqueza producida, en América Latina el $10 \%$ más rico se apropia del $48 \%$ del ingreso total de la región, mientras que el $10 \%$ más pobre apenas obtiene el 1,6\%. En los indicadores de empleo, también se observa un fenómeno regresivo pues la tasa de desempleo en la población económicamente activa crece de 5 \% en 1990 a 11 \% para el 2003 (3).

En este marco de deterioro, la transición demográfica como fenómeno general (aunque no homogéneo) en la región latinoamericana y particularmente el envejecimiento de las poblaciones, hace más complejos los cambios en los patrones de enfermedad y muerte. En América Latina, persisten problemas infecciosos y carenciales, pero además hay un peso creciente de la patología de adultos y de adultos mayores (enfermedades crónicas y degenerativas), lesiones accidentales e intencionales, trastornos psicológicos y adicciones. Emergen padecimientos como VIH/SIDA y nuevas enfermedades como el Síndrome Agudo Respiratorio Severo (SARS)

A pesar de este complejo panorama, los indicadores promedio expresan avances importantes en salud, como la disminución de la mortalidad general, materna e infantil y el consecuente aumento de la esperanza de vida, que llega a 70 años para el año 2000. Sin embargo, las variaciones entre países y al interior de ellos muestran una situación nada halagüeña. La esperanza de vida presenta variaciones de 54 años en Haití a 79 años en Canadá. Las desigualdades en los indicadores de mortalidad infantil también son evidentes. En el año 2000 el promedio para la región de las Américas fue de 24,8 defunciones por mil 
nacidos vivos registrados (NVR), variando de 5,2 en Anguila a 80,3 en Haití y al interior de los países, el rango de variación fue de 3,7 defunciones por mil NVR por año hasta 133 muertes (4).

En este marco, la polarización epidemiológica hace referencia al incremento en las desigualdades de los indicadores de daño, entre países, regiones y grupos sociales, en donde las poblaciones más vulnerables presentan una sobremorbilidad y sobremortalidad por múltiples causas. Mientras que la regresión sanitaria se define como el fenómeno de resurgimiento de enfermedades ya erradicadas o bajo control (5).

Desde nuestra perspectiva, el reconocimiento de los procesos de polarización epidemiológica y de regresión sanitaria es fundamental para entender las condiciones de salud/enfermedad de los latinoamericanos y continuar con propuestas de desarrollo en salud pública.

Además del contexto sociosanitario precedente, entre los referentes particulares del campo de la salud pública, se consideran los siguientes:

Funciones Esenciales en Salud Pública

Constituyen una de las iniciativas más importantes en el contexto de las reformas en salud al final de la década de los 80 , que se desarrolló en el continente con el impulso decidido de la OPS/OMS. La sistematización en once Funciones Esenciales (FESP) da pauta para centrar las acciones en salud pública con un eje ordenador que revalora el papel del estado. Los criterios e indicadores incluidos para su evaluación en la región y los países constituyen un conjunto de parámetros para definir el campo, tanto en la gestión y operación como en el desarrollo de conocimientos (6). Las primeras evaluaciones muestran la escasa atención que han tenido los recursos humanos en salud pública y el desarrollo de conocimientos en este campo, subrayando la importancia que debe tener este tema, que ha sido uno de los grandes ausentes en las políticas de reforma del sector salud (7).

Objetivos y Metas del Milenio (Organización de Naciones Unidas)

Son un referente más, que centra las acciones del poder público en problemas graves que afectan al desarrollo global y más aún a los países y regiones del mundo con menor desarrollo humano y social. Muestran la importancia que le asignan los gobiernos nacionales a la mortalidad infantil y materna, una de las mayores deudas de las sociedades contemporáneas con la población de los países de mayor pobreza. Sin duda intervenir con políticas y acciones orientadas 
a grupos específicos, permitirá reducir las tasas de mortalidad infantil y materna de ese amplio segmento de población; sin embargo, las enfermedades crónicas, emergentes y las características actuales de las poblaciones, también indican los límites de este tipo de acciones en salud pública y plantean retos de mayor complejidad a las sociedades contemporáneas.

Asociación Latino Americana de Educación en Salud Pública-ALAESP

La ALAESP ha desarrollado iniciativas novedosas para mejorar la formación y el desempeño de los recursos humanos en salud pública. Ha formulado contribuciones para la formación de la fuerza de trabajo en salud, para acciones dirigidas a los grupos humanos en situaciones de precariedad, en situaciones de desastre, con la incorporación de visiones analíticas como las perspectivas de clase, género, etnia; o destacar problemas en el ambiente, trabajo, familia; son todas ellas, evidencia de la capacidad de la Asociación. Además, el capital intelectual que representan los directivos de programas académicos, se ve fortalecido por la presencia de funcionarios y gestores de los sistemas de salud, cuya experiencia sintetiza un potencial de creación y transformación muy valioso.

A partir del marco de referencia ya señalado, se ubican tres componentes estructurales para la salud pública: el objeto de conocimiento, la práctica social y la formación de personal.

\section{TRES COMPONENTES}

Objeto de conocimiento

En tanto objeto de conocimiento, la salud pública está inmersa en un devenir histórico que le da identidad y establece los límites de un objeto de la realidad empírica. El desarrollo de sus cuerpos conceptuales se ha nutrido de los aportes de distintas disciplinas, constituyéndose en un campo interdisciplinario.

Su objeto de conocimiento en torno al proceso salud/enfermedad (s/e) y a las formas como la sociedad responde frente a él, ha significado enfrentar distintas posiciones que originalmente tienen su sustento en el campo de la medicina. Si bien la medicina ha nutrido a la salud pública, su avance e independencia conceptual y metodológica hoy día, le otorga una autonomía que le permite contribuir a la comprensión del objeto complejo denominado salud, que involucra distintos aspectos. Una breve referencia a cada uno de ellos permite establecer los retos que se requieren afrontar. 
Niveles: individual, grupal y colectivo

La explicación de las expresiones de salud/enfermedad en los individuos y las poblaciones requiere considerar que en torno a estos fenómenos está indisolublemente articulado el devenir de la existencia humana. Lo que significa referirse a los niveles que van desde lo individual hasta lo colectivo, y que están implicados entre sí; estos niveles hacen referencia al modo como distintos procesos se expresan en la materia orgánica y también en la organización social. Ninguno de estos niveles es suficiente por sí mismo para explicar los complejos procesos que generan la salud y producen la enfermedad. Aquí radica uno de los mayores problemas del abordaje del objeto s/e, no se puede comprender al individuo sin su referente grupal y su vida en la colectividad y en sentido inverso, una aproximación colectiva requiere el reconocimiento de la diversidad grupal e individual para avanzar en la comprensión y eventual transformación de la s/e.

Cada uno de los niveles presenta particularidades específicas y requiere sistemas de análisis y explicación diferentes. El enfoque médico biológico ha tenido un peso preponderante dada su capacidad explicativa en el nivel singular y por los aportes científicos que históricamente ha construido para entender y resolver problemas de salud en los individuos; sin embargo, el traslado mecánico de explicaciones e intervenciones hacia los niveles particular y general, es decir hacia los grupos y las colectividades humanas, ha mostrado su insuficiencia para explicar y generar intervenciones mostrando la necesidad de incorporar otras racionalidades y cuerpos conceptuales.

Dominios: natural y social

El doble carácter natural y social de la enfermedad continúa siendo uno de los aspectos debatidos en la actualidad. ¿Hasta dónde cada uno de ellos es responsable de la producción de los daños en los individuos? ¿Cuál es el tiempo que se requiere para identificar los daños producidos? y una pregunta de mayor complejidad, ¿cómo son los procesos por los cuales se genera y desarrolla el daño?

La epidemiología, como área de conocimiento que aborda la ocurrencia de los daños en poblaciones humanas, ha realizado importantes aportes a partir de su vasta producción científica y del desarrollo de un arsenal metodológico; sin embargo, estos aportes no han sido suficientes y aún son debatidos $(8,9)$; ya que existen múltiples insuficiencias explicativas para dar cuenta de situaciones similares en condiciones diferentes o de situaciones diversas en condiciones semejantes (10). Esas condiciones se perfilan en el dominio social, en donde la 
contribución de las ciencias sociales es aún escasa, los modelos conceptuales de que se dispone son incompletos y las explicaciones no logran permear el arraigado discurso natural biológico; por ello, el debate en torno a la dimensión social, sigue siendo un espacio prolífico para avanzar en propuestas que, sin ignorar los aportes existentes, indaguen en los planos conceptual, metodológico y empírico, para mejorar nuestra comprensión del problema y especialmente para generar propuestas de acción que permitan incidir en los problemas de salud de los grupos humanos.

Disciplinas: biológicas y sociales

La conformación histórica de los cuerpos de conocimiento de las ciencias naturales, ha sido diferencial y la robustez de ellas se explica por su larga existencia temporal, en particular, el desarrollo temprano de la física y posteriormente de la biología. Sin embargo, en el caso de las ciencias sociales, su desarrollo histórico es más reciente y las teorías construídas, al reflexionar sobre lo humano, son dinámicas y expresan la incertidumbre de las transformaciones de las sociedades, por lo que frente a las regularidades, certidumbres y respuestas unívocas de las ciencias naturales del discurso científico dominante, quedan en franca desventaja.

Sin embargo, a pesar de esa diferencia (y distancia) entre las disciplinas biológicas y sociales, éstas últimas son hoy día un referente insustituible para la comprensión y explicación de los problemas colectivos de salud y para las acciones públicas de intervención. Para la salud pública forman parte ya de su cuerpo de conocimientos, tanto para la formación como para la práctica social de las profesiones que en ella participan.

Temporalidad: retrospectiva y prospectiva

La comprensión de los problemas de salud y de las prácticas sociales disponen del tiempo como una categoría de análisis tanto para identificar los procesos, como para comprender su variabilidad. La mirada hacia el pasado es el recurso conceptual y metodológico más significativo que se ha usado. Es justamente en la expresión temporal de los fenómenos en donde radica una de las fuentes de veracidad que sustentan múltiples conocimientos y justifican muchas de las explicaciones de la salud pública. Sin embargo, la necesidad inevitable de conducir las acciones en el presente con una mirada de futuro ha dado pauta para que se desarrollen otro tipo de propuestas, especialmente en el campo de la planeación. 
Así, en el campo de la administración, gestión y planificación de los sistemas y servicios de salud, este posicionamiento de mirar al futuro ha logrado un desarrollo importante, desde los aportes instrumentales de la planificación de servicios, hasta los ejercicios prospectivos más recientes (11). Aún con la falta de certeza, es indudable que el accionar en salud pública no puede prescindir de la posibilidad de anticiparse a eventos diversos, ya que está en su esencia fundacional, colocarse antes de la enfermedad, controlar la naturaleza provocadora de daños y garantizar seguridades futuras.

El desarrollo de planes, programas y acciones en salud pública ante riesgos potenciales, ha incorporado con éxito conjuntos de intervenciones y su capacidad de respuesta ha tenido resultados positivos para la salud y la vida de las personas, fortaleciendo y legitimando un quehacer sanitario por fuera y antes de la enfermedad (12).

Dimensiones: singular, particular y general La comprensión de la salud-enfermedad en sus diferentes manifestaciones, requiere su valoración en distintas dimensiones, que sin ser excluyentes, si requieren de estrategias y abordajes conceptuales y metodológicos distintos. Lo singular no solo alude al individuo aislado, sino principalmente al proceso o conjunto de procesos que aunque exprese circunstancias similares con otros sujetos, le es propio y característico, con capacidad de generalización sólo aplicable a ese conjunto de sujetos singulares.

Lo particular, remite a un nivel intermedio de los procesos, que tienen lugar en un aquí y ahora definido, con causas y consecuencias aplicables a una gama mayor de similitudes y poca diferenciación, al menos no con la suficiente fuerza como para ser asignada a otro conjunto de situaciones y procesos. Conserva de lo singular los rasgos de los integrantes del conjunto y expresa simultáneamente las características de la generalidad; siendo así el nexo mediador entre los procesos generales y los singulares.

Por último, el nivel de lo general alude a un plano de análisis que sitúa los procesos en su capacidad explicativa para una amplia gama de conjuntos, sin que tenga el detalle suficiente para determinar los movimientos específicos que ocurren en lo singular, pero si con capacidad de determinación a ellos bajo las circunstancias específicas que señala lo general. 
Problema complejo

La conclusión que se deriva de las consideraciones precedentes, es que para lograr avances en el conocimiento de la salud/enfermedad, es necesario interpretarla como un problema complejo (13). Tanto por las características que presenta en sus manifestaciones empíricas, como por los modos como se puede realizar la aproximación a su conocimiento y comprensión.

Incorporar distintas perspectivas científicas, ubicarla en distintos niveles de existencia, de expresión y de interpretación disciplinaria, supone un esfuerzo de abstracción complejo que adquiere aún mayor relevancia al posicionarnos desde la salud pública.

Así, desde la salud pública nos enfrentamos a dos situaciones. Por un lado, la ubicación de los problemas de salud en múltiples planos de la realidad a partir de su naturaleza compleja, donde el recorte de las implicaciones, atributos y circunstancias que inciden y determinan al objeto no es una simple abstracción de la realidad, es esencialmente un proceso de razonamiento donde la realidad material se convierte en el criterio de verdad sobre el propio objeto. Por el otro, se opera con un conjunto de referencias conceptuales y metodológicas que no se limitan a la adopción de teorías y conceptos establecidos en otros cuerpos disciplinarios o en la misma salud pública, sino que requiere esencialmente un proceso de traducción, adaptación, reinterpretación de los conceptos y teorías. Con una necesaria vigilancia de su racionalidad y estructura epistemológica, conceptual y metodológica, al mismo tiempo que se hace una reinterpretación a la luz del objeto situado en la realidad.

Práctica social

En tanto práctica social, la salud pública es un escenario donde confluyen áreas profesionales típicas de la atención a la enfermedad como las profesiones de medicina, enfermería, odontología, psicología; pero también otras, como la administración, ingeniería, veterinaria, economía y sociología. Esto hace un conjunto donde tradicionalmente la hegemonía se ejerce desde la medicina, pero ésta no puede reclamar exclusividad.

Así, es posible distinguir más allá de las profesiones concretas, un conjunto de prácticas profesionales que son específicas de la salud pública, lo que genera un nuevo entramado científico-técnico que adquiere reconocimiento social, en donde la experiencia dominante en el mundo, es que la salud pública conforma una práctica social que requiere de una formación de posgrado (14). 
- Público Estatal: sistemas, instituciones, servicios, prácticas

La salud pública tiene desde su origen un adjetivo que alude al ejercicio desde la estructura del poder público, por lo que marca distancia de las prácticas profesionales privadas, aun cuando puede incluirlas. Lo privado no es un atributo distintivo de la salud pública, incluso en aquellos países donde las reformas del sector imprimen un sesgo privatizador a todo el sistema sanitario.

La acción desde el Estado, implica que la salud pública se ubique en el terreno de los bienes públicos. Los espacios donde adquiere existencia y relevancia son estructuras del Estado, ya sea en los niveles nacionales, regionales o locales; tanto en niveles normativos, como operativos. ¿Cómo imaginar un ejercicio de la salud pública sin apoyo en funciones estatales? Incluso para autores como E. Granda, la fuerza del Estado a través de las acciones públicas para controlar la enfermedad, forman parte del "trípode fundacional" de la salud pública (15).

Las instituciones de salud, han surgido desde un cierto modelo hegemónico de conocer y actuar en salud pública. En estas instituciones se cristaliza una interpretación de la responsabilidad del Estado que norman, validan y legitiman prácticas en salud pública desde una perspectiva técnico-política y conforman modelos de práctica social.

- Práctica general de colectivos

La salud pública es una práctica que técnicamente se formaliza en las instituciones educativas y se realiza en los sistemas, instituciones y servicios de salud; sin embargo, su vigencia no se limita a estos espacios, sino que incluye las interacciones con los individuos que conforman los colectivos humanos a quienes van dirigidas sus acciones. En estos colectivos se expresan los resultados positivos y negativos de las intervenciones, pero sobre todo, producen un conocimiento experiencial, pues las poblaciones se apropian de esas prácticas, conservándolas, transformándolas, adaptándolas e incluso rechazándolas. En este tránsito, pasan a formar parte de las prácticas sociales de los colectivos humanos y son entonces, prácticas generales de la sociedad sobre la salud pública. Como práctica social, la salud pública está internalizada en todos los integrantes de la sociedad, que pueden realizar acciones individuales o colectivas para preservar la salud y evitar la enfermedad. De este modo, se trata de la pertenencia y conocimiento social de la salud pública que escapa al dominio y control técnico científico de los profesionales de la salud pública. 
- Práctica profesional de grupos

El reconocimiento técnico y científico adquiere madurez con la profesionalización, en el caso de la salud pública su diferenciación ha sido como una especialización técnico-científica de posgrado. Pero la caracterización de los atributos específicos que la definen es una tarea inconclusa.

Pueden ubicarse tendencias dominantes y allí la medicina ha tenido preponderancia, pero no ha sido la única, pues muchos de los aportes han sido resultado de prácticas específicas de otras profesiones.

Lograr identificar las prácticas en salud pública que realizan los distintos profesionales, es una necesidad que permitirá establecer las competencias necesarias a desarrollar y en consecuencia, definir con mayor nitidez lo que deben hacer y las habilidades que deben tener dichos profesionales.

Formación de personal

La inserción institucional de la formación académica de los posgraduados en salud pública, corresponde a espacios académicos universitarios y por ello no pueden sustraerse a la dinámica que imprime la lógica de esas instituciones; y tampoco pueden aislarse de la dinámica de los sistemas e instituciones de salud, ya que es allí donde tienen su función social y adquiere concreción la práctica profesional. Así una referencia central para la formación y práctica está en el concepto de salubristas del futuro como mediadores estratégicos (16), en los que la competencia técnica y el compromiso con procesos amplios e incluyentes de construcción de agendas públicas por la salud y la vida digna, supone un compromiso ético.

En consecuencia, la tarea de construir redes de interrelación entre los espacios académicos y los ámbitos de servicio, así como entre éstos y las colectividades, demanda fortaleza conceptual, diversidad metodológica, competencia técnica, creatividad y compromiso ético, en la perspectiva del sanitarista como decodificador de la salud (problema complejo) y mediador estratégico entre múltiples actores.

- Profesionales

La formación de profesionales alude fundamentalmente al posgrado, ya que la licenciatura en casi todos los casos se refiere a profesiones como medicina, odontología, enfermería, nutrición, sociología, administración, 
economía. Es entonces en el posgrado donde tenemos la mayor intervención en la formación de profesionales en salud pública.

Las dificultades que hay que sortear, también son múltiples y remiten a distintos aspectos, entre los que destacan: la orientación hacia la investigación o el énfasis en el desempeño de los sistemas, instituciones y servicios de salud; o una combinación de ambas. Definiciones que implican cuerpos de conocimientos específicos, procesos pedagógicos y modelos de formación. Cabe destacar - por su importancia - las acciones que en torno a la calidad de la educación de posgrado han realizado la OPS y la ALAESP y su aporte significativo para la formación (17).

\section{- Técnicos}

Los técnicos son parte de la fuerza de trabajo en salud con relevancia en las acciones institucionales. Por lo tanto pensar en su formación y capacitación resulta un componente importante de considerar. Las experiencias nacionales y las circunstancias históricas del surgimiento de los técnicos en salud, aportan experiencias valiosas. El caso más significativo es la lucha contra la malaria y el dengue, sostenida con la participación de técnicos en acciones de salud pública.

\section{- Formación profesional}

Dentro de las modalidades de formación podemos distinguir la formación profesional, como aquella que otorga grados académicos con reconocimiento legal, pero no únicamente, pues aún cuando la formación de posgrado es necesaria, también es importante incidir en los niveles de licenciatura con aspectos relevantes de la salud pública para que los profesionales amplíen su visión sobre el campo de la salud y no sólo se limiten a la acción curativa, ni a la atención individual.

\section{- Capacitación y actualización}

Tanto para profesionales como para técnicos, la formación continua y la actualización son una necesidad. Ya sea la implantación de programas específicos, o porque el conocimiento actual impone nuevas formas para afrontar los problemas o porque los procesos de calificación o ascenso laboral lo requieren. En todo caso la capacitación y actualización, son un conjunto de prácticas educativas que inciden en la formación de personal de salud pública y que constituyen una responsabilidad a cumplir. 


\section{LOS RETOS}

Para la Asociación Latinoamericana y del Caribe en Educación en Salud Pública los próximos años estarán definidos por el reconocimiento de las iniciativas y acciones desarrolladas en años precedentes que, mirando hacia el futuro, deberá incorporar actividades que permitan la confluencia con la OPS/OMS y otras organizaciones con quienes ya mantiene un vínculo sólido. Las vertientes que se señalan a continuación, marcan esta tendencia.

- Intervención en la formación:

Continuar con la formación de trabajadores en salud pública, tanto en la educación académica (especializaciones, maestrías, doctorados) como en educación continua y perfeccionamiento. Avances importantes con antecedentes en varios países son:

- Definición de competencias para la fuerza de trabajo en salud pública. Establecer referentes generales como marco para todos los programas educativos, será sin duda una contribución significativa.

- Campus Virtual en Salud Pública.

Se constituye en un recurso con resultados inmediatos, al usar plataformas educativas que nos acercan a todos, permite el intercambio y ofrece soluciones a carencias generales o puntuales.

- Calidad de la educación en salud pública

Desarrollo de un conjunto de propuestas para mejorar la acción educativa, más aún si se considera que existen varias experiencias nacionales (Brasil, Colombia, México) en la acreditación de programas de posgrado en salud pública, por lo cual es posible crear un sistema de acreditación regional que permita ubicar la calidad desde la perspectiva de la Asociación.

- Construcción de conocimientos

Sin duda el apoyo que la OPS/OMS ha brindado, resulta valioso para continuar desarrollándolo con la participación de programas educativos en proyectos multicéntricos sobre temas relevantes. Aquí, las contribuciones teóricas, metodológicas y técnicas de los investigadores de América Latina y el Caribe, requiere de mayor visibilización

- Práctica Profesional

La caracterización de las formas que adopta la práctica profesional en los diferentes países y en la región es necesario, ya que se podrá tener 
un marco de situación para incidir en fortalecer este campo, y al mismo tiempo desarrollar referentes para el diseño curricular. Destacan por su importancia la identificación del mercado laboral y de buenas prácticas de salud pública con autonomía de otras profesiones.

Para finalizar, la ALAESP requiere acciones organizativas para incorporar a los nuevos programas que existen en la región. La organización interna que dinamice la participación y ofrezca cobertura en un marco de autonomía y respeto a las subregiones, países y programas, la ubicará en una posición de liderazgo y fortaleza frente a los otros actores del campo de la salud pública

\section{REFERENCIAS}

1. Granda E. Formación de salubristas: algunas reflexiones. En: La salud colectiva a las puertas del siglo XXI. $1^{\text {a }}$ edición. Medellín: Universidad de Antioquia; 2000.

2. Banco Mundial. Informe sobre el Desarrollo Mundial 2004. Washington, D.C: Banco Mundial/Oxford University Press; 2003.

3. La Jornada, México, 25 de abril, 2004, p. 43. Datos reportados en la reunión anual del FMI.

4. Organización Panamericana de la Salud. Informe de Salud de las Américas 2002.Vol. 1.Washington, D.C: OPS; 2003.

5. López O, Blanco J. Los retos en salud para el fin de siglo. En: Gutierrez E (coord). El Debate Nacional. 5. La Política Social, México: Editorial Diana; 1997.

6. Organización Panamericana de la Salud. La salud Pública en las Américas. Nuevos Conceptos, Análisis del Desempeño y Bases para la Acción, Washington, D.C: OPS; 2002.

7. Homedes N, Ugalde A. Why neoliberal health reforms have failed in Latin America. Health Policy. 2005; 71:83-96.

8. Breilh J. Epidemiología crítica. Ciencia emancipadora e interculturalidad. Buenos Aires: Lugar Editorial/Universidad Nacional de Lanús; 2003.

9. Almeida N. La ciencia tímida. Ensayos de deconstrucción de la epidemiología. Buenos Aires: Lugar Editorial, Universidad Nacional de Lanús; 2000.

10. Luz M. Natural, racional, social. Razón médica y racionalidad científica moderna. $1^{\text {a }}$ edición, Buenos Aires: Lugar Editorial; 1997.

11. Testa M. Pensamiento estratégico y lógica de programación (El Caso de la Salud). $1^{\text {a }}$ edición, Buenos Aires: Lugar Editorial; 1995. 
12. Jarillo E, López O, Mendoza JM. La formación de recursos humanos en salud pública. En: Vásquez D, Cuevas L, Crocker R (coords.) La formación de personal de salud en México. México: Programa colaborativo de recursos humanos en salud, OPS; 2005.

13. Morin E. Introducción al pensamiento complejo. $1^{\text {a }}$ edición, Barcelona: Gedisa; 1994.

14. Borrel RM. La educación médica de postgrado en Argentina: el desafío de una nueva práctica educativa. $1^{\text {a }}$ edición. Buenos Aires: OPS; 2005.

15. Granda E. la salud pública y las metáforas sobre la vida. Revista de la Facultad Nacional de Salud Pública. 2000; 18(2): 83-100.

16. Granda E. ¿A quéllamamos salud colectiva hoy?. Revista Cubana de Salud Pública. 2004; 30(2): 23-43.

17. Organización Panamericana de la Salud. La calidad de la educación en salud pública: un desafío impostergable. Serie de Recursos Humanos No. 32. Washington: OPS;2002. 